\title{
Danblon, Emmanuelle. 2013. L'homme rhétorique (Paris : Cerf/Humanités)
}

\section{Roselyne Koren}

\section{(2) OpenEdition}

1 Journals

\section{Édition électronique}

URL : http://journals.openedition.org/aad/1688

DOI : $10.4000 /$ aad. 1688

ISSN : 1565-8961

Éditeur

Université de Tel-Aviv

\section{Référence électronique}

Roselyne Koren, « Danblon, Emmanuelle. 2013. L'homme rhétorique (Paris : Cerf/Humanités)», Argumentation et Analyse du Discours [En ligne], 12 | 2014, mis en ligne le 20 avril 2014, consulté le 22 septembre 2020. URL : http://journals.openedition.org/aad/1688; DOI : https://doi.org/10.4000/aad. 1688

\section{Ce document a été généré automatiquement le 22 septembre 2020}

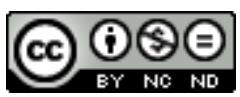

Argumentation \& analyse du discours est mis à disposition selon les termes de la licence Creative Commons Attribution - Pas d'Utilisation Commerciale - Pas de Modification 4.0 International. 


\title{
Danblon, Emmanuelle. 2013. L'homme rhétorique (Paris : Cerf/ Humanités)
}

\author{
Roselyne Koren
}

\section{RÉFÉRENCE}

Danblon, Emmanuelle. 2013. L'homme rhétorique (Paris : Cerf/Humanités) 226 p., ISBN :

9782204099264

1 La fonction persuasive (Danblon 2005: 7) avait l'acte de persuader pour objet ; L'homme rhétorique est essentiellement consacré à une seconde fonction tout aussi essentielle de l'activité rhétorique: "raisonner », et aux diverses strates de la raison humaine. Il s'agit certes d'un ouvrage scientifique : l'auteure, Emmanuelle Danblon, est professeur de rhétorique à l'Université libre de Bruxelles, l'ouvrage comprend un appareil de notes rigoureux et éclairant, qui enrichit la démonstration et donne de nombreuses références bibliographiques. Mais le lecteur se trouve en fait invité à lire un essai qui a des accents de manifeste, un ouvrage convaincu qui allie argumentaire expert et apologie passionnée d'une conception « naturaliste » et « humaniste » de la rhétorique.

2 L'auteure a pour ambition de dépasser les clivages entre rationalité théorique et pratique, raison, passion et émotions, corps humain, sensations et entendement, raisonnement logique formel et pratiques discursives de figuration. Il s'agit de revisiter et de reconfigurer la notion de technè et d'explorer la fonction de "passeur » de la rhétorique - "passeur entre les disciplines ", " outil de formation des futurs citoyens » et « garde-fou humaniste contre les dangers constants qui guettent la raison humaine : le dogmatisme et l'obscurantisme » (23). La rhétorique est présentée tout au long de la démonstration comme l'une des facultés à la fois innées et acquises de la nature humaine, comme l'une des formes fondamentales que revêt l'intelligence humaine. Mais elle est aussi un «art de vivre» (62), et la condition de possibilité de la liberté 
humaine, de la distance critique et de la prise de conscience éthique. Danblon propose donc une "vision élargie, plus cohérente et plus réaliste de la raison humaine» (10) qui permet de penser la rhétorique non plus comme une somme de techniques auxquelles chacun de nous serait libre ou non de recourir, mais comme une faculté universelle de " cet Homo rhetoricus, artisan de la cité, maître du logos » qui habite chacun de nous et nous permet de figurer ce qui fait sens pour nous. L'homme rhétorique se doit de trouver dans le monde complexe et imprévisible où nous vivons - à la force du poignet, de ses « facultés » et de ses « décisions » (10) - des solutions à des dilemmes existentiels « hors de notre mesure » (60).

3 Il s'agit donc de démontrer qu'une "cascade de dichotomies"(42) ou de "grandes oppositions héritées à propos de la raison » (38) empêchent d'évaluer la rhétorique à sa juste valeur et de penser l'homme rhétorique comme un être complet. Il s'agit des "bases biologiques de la raison" soit l'opposition entre le corps, "véhicule » des «sensations» et «l'entendement»; de la « césure entre raison et émotions»; de la «concurrence entre rigueur et souplesse » en matière $d$ ' « intelligence humaine »; de l'opposition des qualités attribuées au «langage écrit» et au «langage oral»; de la césure entre discipline " pratique » dévalorisée, jugée irrationnelle (38-42) et discipline "théorique " dont la nature garantirait automatiquement la rationalité. Face à ces clivages le plus souvent présentés comme inconciliables, Danblon va tenter de proposer et de justifier une conception "continuiste" (39) dont l'originalité est due au positionnement suivant: "la rhétorique est présente dès les premières sensations corporelles jusqu'aux projets intellectuels les plus ambitieux » (42); elle sera conçue comme «entière " et "humaniste " et non pas comme «réductionniste ni irrationaliste ». L'auteure pratique donc, entre autres, à cette fin ce que Charaudeau (2010) qualifie d'«interdisciplinarité focalisée». Il s'agit de l'intégration et de la reconfiguration, dans la théorie de la rhétorique, de concepts empruntés aux "neurosciences", aux «sciences cognitives", à «l'anthropologie» et à la «neurophysiologie » (60) bref, il s'agit d'œuvrer avec ces sciences à une "vision plus complète de l'homme» (154-155) qui inclut, par exemple, la «base biologique de l'abduction et ses sauts intuitifs ».

4 Les qualifications "prolongement» (14), «continuité » (32-33), « démarche généalogique » (58-59), « raison stratifiée » $(112,125-126,147)$ ont donc pour fonction d'unifier trois types d'activités humaines en interaction: la rhétorique spontanée et naturelle de l'homme rhétorique "premier artisan du logos», " humain aux mille tours », aux « mille ruses » $(9-10,13)$; la transformation de cette capacité en « art » à enseigner et à exercer; puis la théorisation de cet art. Il s'agit donc de démontrer que la rhétorique sous toutes ses formes est «l'une des grandes expressions de la culture humaine comme prolongement de sa nature. [...] L'exercice de la rhétorique est à la fois le propre de l'homme et une condition de son humanité » (10). En résumé :

5 L'esprit de la rhétorique, dans toute sa raison pratique, consiste précisément à fréquenter de façon dynamique les différentes strates de la raison humaine, comme on fréquenterait quotidiennement tous les étages d'une grande maison, y compris le grenier et la cave qui souvent recèlent de très anciens trésors (125-126).

6 Il s'agit pour Danblon, dans l'introduction, de justifier le choix d'un angle d'attaque «naturaliste » en matière de rhétorique, soit de dépasser la réduction de "la raison à sa biochimie » comme le refus « de comprendre l'esprit à partir de la matière » (14). Le rhétoricien se doit de redevenir aristotélicien et de «replacer l'homme, cet animal 
politique, dans son milieu naturel : la culture » (ibid.). La première partie de l'ouvrage est donc consacrée à la question: "comment l'histoire consciente du logos a-t-elle débuté ? Et comment distinguer entre une "intelligence de réaction devant l'imprévu » attribuée à l'« habileté », à l'« ingéniosité » humaines, et l'accusation de «fourberie » ? (15). La démonstration sera donc consacrée tout d'abord à la "rhétorique spontanée " forgée dans une culture "artisanale» ou culture du "savoir-faire", rhétorique illustrant la créativité et l'intelligence d'une «raison pratique» fondatrice. La rhétorique "technique» en est issue, elle émergera dans un second temps, "au moment où s'opère le grand changement sociétal qui a vu se techniciser la rhétorique " (16). L'accent est mis sur la «force de l'homme rhétorique des origines, une force qui est le lieu de sa vitalité », vitalité " du vivant » qui veut «tailler le monde à sa mesure " (20). Juger, décider, célébrer et témoigner sont des activités rhétoriques vitales qui seront décrites dans leur "dimension de rhétorique naturelle et technique » (ibid.). La pertinence et la validité de ces descriptions seront réexaminées et discutées à la fin de l'ouvrage au prisme d'une étude de cas - le témoignage d'une rescapée de la Shoah, enregistré par la Fondation Auschwitz à Bruxelles, pour mettre les hypothèses «à l'épreuve de faits empiriques " (23). Cette analyse critique sera suivie d'une conclusion "en forme d'ouverture", qui tentera de répondre à une question simple mais essentielle : « que peut la rhétorique aujourd'hui ?» (23)

Le premier chapitre revisite l'histoire de la création de la rhétorique au prisme de la conception naturaliste et humaniste défendue par l'auteure et souligne d'emblée que " dès le départ, avec la rhétorique, il y a problème. Quand est-elle née ? Quelles sont ses origines? " (25) Est-elle avant tout « divine », « mythique » ou «logique », « le propre de l'homme ", " matrice de démocratie » ou de " tyrannie ", " garantie pour la liberté » ou «bras armé des propagandes»? Ce chapitre a donc pour fonction essentielle de problématiser le «brouillage » des origines de la rhétorique, brouillage qui débouche sur un paradoxe à résoudre et à dépasser: «la rhétorique est partout dans la vie publique mais elle est très rarement pensée consciemment en tant que telle par ses usagers que sont les citoyens » (28). Danblon entame ainsi " une généalogie de la raison humaine, en tant qu'elle est rhétorique ». L'enjeu de cette démarche est la tentative de répondre aux questions « Qui est cet homme rhétorique? Que fait-il lorsqu'il fait de la rhétorique ? ». Comment la "puissance ingénieuse de l'homme », confronté à la "force sauvage de la nature » se transforme-t-elle en une technique lui permettant de figurer le monde à sa mesure? Ce questionnement se refuse à préjuger au départ de la réponse et affirme vouloir "se nourrir de tous les champs du savoir qui pensent la raison humaine : la linguistique et l'anthropologie, le droit et la philosophie, la psychologie et les sciences cognitives » (30). L'analyse des points de vue platonicien et aristotélicien occupe certes une place centrale, mais Danblon accorde une place tout aussi cruciale à quelques penseurs qui abondent au cours des siècles dans le sens d'Aristote et contribuent à la critique du clivage et des dichotomies entre les différentes strates de la raison humaine: Vico, Nietzche, Aubenque, Berthoz, Kennedy, etc. (42-62). L'épilogue de cette histoire de la « résistance » aux « cascades de dichotomies » a pour fin d'« éclairer la situation actuelle » et plus particulièrement le fait que la question du statut de la rhétorique est même "redevenue d'une brûlante actualité » depuis les années 1960. Et de souligner que personne sans doute mieux que Chaïm Perelman n'a exprimé ce «renouveau » dans la Nouvelle Rhétorique, ouvrage qui s'inspire de l'œuvre d'Aristote, la reconfigure et s'oppose radicalement au rationalisme cartésien afin de revisiter et de fonder la raison pratique rhétorique (63). La rhétorique n'est donc pas 
conçue ni présentée comme une "théorie", mais comme un ensemble de pratiques « au service d'une société d'hommes dont la rationalité est d'abord dans l'action » (67). La contestation du clivage entre raison théorique et raison pratique passe ici par l'affirmation suivante: "Fondement ou base aux constructions intellectuelles les plus élaborées, le corps des hommes n'est pas l'ennemi de leur 'esprit' : il est leur esprit » (ibid.).

Le second chapitre " est consacré aux diverses fonctions de la rhétorique ». Il tente de démontrer ce qu'est "penser en action", "exprimer ses émotions, raconter des histoires », activer "l'intelligence pratique de l'artisan » qu'est l'homme rhétorique dans un premier temps (70). Mais il s'agit ensuite de définir la seconde strate, celle de la "rhétorique technique», prolongement de la "naturelle» (126), soit cet "art [ou tèchnè], issu de l'ingéniosité humaine " qui conduit l'homme «à utiliser sa nature, à en développer les fonctionnalités » (69). C'est ici que Danblon développe l'un des concepts centraux de l'ouvrage, "la figuration». Il réfère aux activités rhétoriques de la représentation ou configuration des réalités auxquelles l'homme se mesure et qui font sens pour lui. «Le tour du potier, la figure de style de l'orateur, la barre assurée du navigateur, le geste sûr du médecin », sont " autant de réponses pratiques, humaines, intelligentes, à la complexité du réel » (61). "L'art rhétorique » ou technè se compose d'une «liste infinie et toujours ouverte» d'activités nécessaires à la vie sociale : délibérer, décider, juger, conseiller, célébrer, déplorer, condamner ou louer, témoigner, réconcilier, transmettre, critiquer (77), activités qui le contraignent à «fréquenter toutes les aptitudes de son intelligence pratique» (84). L'une des illustrations emblématiques de l'activité de figuration est le genre épidictique, «le plus paradoxal des genres de la rhétorique, le plus 'émotif' et le moins 'décisionnaire' des trois genres, le plus rhétorique mais le moins argumentatif» (98). Sa fonction principale est le rétablissement ou le maintien de la concorde, l'équilibre social (95), l'éveil d'une « disposition à l'art de vivre ensemble » (98). La figuration y apparaît donc comme une "fonction vitale ", comme la condition de possibilité de la "raison humaine ", comme une "compensation", une "consolation" au devoir de délibérer et aux risques de dissensus qu'il entraîne" (99).Les artifices de la rhétorique technique viendront prolonger, en l'occurrence, les figures d'une rhétorique naturelle, "régulatrice d'un certain rapport au monde ». Le développement de la rhétorique technique constituera ainsi « une réponse rationnelle à l'inquiétude naissante face aux discordes potentielles nées de la nécessité de délibérer » (100). En se technicisant la rhétorique se divise en genres : « il y a un temps pour critiquer, juger et délibérer. Il y a un temps pour réunir et assurer la concorde » (ibid.) et tenter de donner un sens commun au monde (101). Le genre épidictique est donc, psychologiquement, le plus émotionnel des genres rhétoriques, et, éthiquement, le plus juste. Le monde épidictique est "éthicoesthétique, dans sa formulation, comme dans ses principes. [...] Comme 'passeur', l'épidictique est le lieu, la forge de l'artisan, où toutes les figurations s'élaborent, des plus intimes aux plus collectives» (201).

9 L'exploration de l'«édifice stratifié » de la raison humaine passe ensuite de la figuration des émotions à celle des événements : leur « mise en récit » est à l'ethos ce que «l'expression des émotions est au pathos ». L'ethos et le pathos sont des «moteurs pour l'action» (104). La conception naturaliste du récit que défend ici Danblon la conduit à en inverser le statut. Il n'est plus perçu comme un genre discursif descriptif et marginal, mais comme le prolongement du mythos par le logos (105). La rhétorique naturaliste est ici confirmée précisément par les sciences cognitives et par les résultats 
de la recherche généalogique s'interrogeant sur l'origine du langage (ibid.). Bref, la variété des aptitudes cognitives du récit est à peu près infinie : « de l'ordonnancement du monde à l'ouverture d'esprit, en passant par la souplesse du raisonnement et la construction de l'identité, individuelle et collective » (125).

L'auteure a tenté d'explorer le lien qui lie la rhétorique technique à la rhétorique naturelle, dans le cas de la pensée en action, de l'expression des émotions et de la narration. Le développement suivant est consacré, en revanche, à un palier différent et distinct de l'«édifice rhétorique»: la suspension des jugements, technique entièrement construite dans le logos afin de "répondre à un nouveau besoin institutionnel, lié à la démocratie naissante : l'obligation de délibérer » (126-127), la nécessité de convaincre "partout où les hommes doivent faire des choix ». Cet outil «inverse " la tendance naturelle de l'homme rhétorique, il ne la prolonge pas: ce dernier doit être capable de " critiquer » sans « détruire ", soit d'observer et d'analyser, sans les " célébrer » ni les "condamner », des opinions différentes des siennes. Tout point de vue devra donc être considéré a priori comme "également raisonnable ». On entre dès lors dans l'ère critique des « discours doubles » ou dissoi logoi, qui ne prolonge pas les traditions « massivement acritiques » des "premières sociétés orales » (129). Il s'agit d'habituer l'esprit à « une plasticité mentale qui n'est ni naturelle ni pathologique mais qui est un acquis de la technique ", l'aptitude à la critique $(132,134)$ qui nous apprend à penser à rebours de nos choix spontanés. Il y a ainsi éducation à l'ouverture à l'autre et créativité, soit développement des « capacités à résoudre des problèmes et à imaginer des solutions » (135). «Penser en action et agir en pensant » sont ici les deux faces d'un même trait d'intelligence; "à défaut d'être linéaire, une telle logique est efficace et intelligente", soit rationnelle (140). Elle ne doit pas être confondue avec la « célébration » ni la « condamnation »; il s'agit d'un «travail de taille » du point de vue du sujet qui utilise la confrontation avec celui de l'Autre ou entre points de vue différents du sien pour mieux se connaître et se préparer à l'action dans la vie politique et sociale. «Dans un monde ouvert, une idée n'est jamais mieux éclairée que par son ombre portée » (145).

11 La pratique de la suspension du jugement conduit nécessairement à la question de savoir comment décider (148); la concurrence entre raison théorique et raison pratique est ici plus centrale que jamais : «celui qui décide est-il guidé par le calcul logique ou par l'intuition? En passe-t-il toujours par une délibération en utilisant la critique, ou pratique-t-il les sauts intuitifs pour produire une persuasion qui livrera le passage à l'action?» (149) La démonstration tente de prouver qu'en dépit de l'oscillation entre ces contraires, la réalité des usages ne prévoit « aucune dichotomie de ce genre ». "L'homme rhétorique utilise spontanément sa raison pratique, sans tergiverser à l'infini »; ses facultés naturelles sont prolongées et renforcées par la technique et réciproquement, la rationalité, la justesse et l'efficacité de ses décisions sont à ce prix (169). Ceci conduit Danblon à revisiter le point de vue d'Aristote, auteur de la Rhétorique, mais surtout en l'occurrence de l'Éthique, au prisme des «acquis récents des domaines qui s'intéressent à la décision » (ibid.) et plus particulièrement des sciences cognitives, de la neurophysiologie contemporaine qui nous donne une « représentation du cerveau comme un simulateur d'action » (154). L'argumentaire de Danblon est donc bien toujours celui d'une rhétoricienne : son regard sur la biologie de l'être humain n'a d'autre but que de «replacer l'homme dans son environnement 
complet, en tenant compte de toute sa rationalité et en cherchant à voir ce que les techniques qu'il a inventées par la rhétorique apportent à sa nature première » (155).

Le lecteur bénéficie ici d'une remarquable démonstration des enjeux épistémologiques de l'interdisciplinarité et d'une interprétation innovatrice de la notion rhétorique d'«homme complet " confronté à l'indéterminisme et aux incertitudes du monde moderne. La nécessité de trancher rend la fonction de la tèchnè d'autant plus cruciale. Danblon expose ici les tenants et aboutissants de la praxis ou art de la délibération qui se doit de déboucher sur la situation de décision ou poièsis où l'homme rhétorique devient le "protagoniste de sa vie " et apprivoise l'incertitude $(153,160)$. L'analyse critique du rapport à la décision de Platon et Aristote à nos jours permet ainsi d'affirmer qu' « une culture entière se niche derrière toute conception de la décision ». Notre conception de la "prudence", comme guide de l'action morale, aura donné naissance à «de nouveaux principes: le 'principe de précaution', le 'principe responsabilité', le 'risque zéro' » (163).

13 La procédure menant à la décision est étroitement liée à celle du jugement qui nous place concrètement face à l'un des plus grands défis de la raison pratique : " par quels moyens, par quel chemin emprunté, un individu se trouve-t-il en situation de juger?» (169) La méthode choisie ici est l'analyse du récit d'une histoire vécue, du témoignage d'une rescapée de la Shoah, en provenance des Archives de la Fondation Auschwitz de Bruxelles. Danblon l'a choisi car il constitue à ses yeux «un réel défi pour la rationalité ». Confrontée à son arrivée à Auschwitz à une situation invraisemblable "qu'aucun exercice de sophiste n'aurait pu inventer " (171), la déportée démontre par le récit de ses réactions, le caractère inductif et non pas déductif d'un jugement constituant l'étape finale d'un parcours où elle est passée tour à tour et spontanément par toutes les strates de la raison pratique. C'est à l'aune de l'« efficacité » de cette démarche, puis du «statut rationnel» de ce jugement et de la question de l'« universalité » (170) que l'inversion de perspective à laquelle la culture rhétorique nous enjoint est analysée. La déportée passe en effet de l'impossibilité de figurer ce qu'elle voit à une démarche de "déni » où l'auteure retrouve quelques-unes des fonctions du genre épidictique: "styliser le monde ", tenter de projeter sur lui une vision des choses alternative consolatrice où il est possible de donner du sens au cours des choses (173). Le déni est ici une «ressource de survie» (174), un acte efficace puisqu'il permet de résister à la réalité « invraisemblable » du camp d'extermination. La déportée quitte ensuite Auschwitz: elle est transférée dans un camp de travail à Berlin où elle "passe du statut de victime à celui de travailleuse » (175). C'est alors qu'elle parcourt toutes les strates évoquées dans les chapitres précédents : adaptation spontanée à une situation active entièrement nouvelle, sensations jamais éprouvées qui lui permettent de reconfigurer son identité (175-176), travail physique mécanisé qui lui permet de laisser son esprit vagabonder et tenter de mieux comprendre ce qui lui est arrivé, introspection stimulée par l'action physique, prise de conscience et mise en mots d'un sentiment de honte, figuré par des métaphores: "elle se sent ", précise Danblon, "comme dans une 'boule', laquelle l'inonde d'une sensation 'rouge' et 'gluante'. Cette figuration par une couleur et par un toucher se traduit chez le témoin par [...] une émotion éthique : la honte » (178). Cette émotion la conduit à un sentiment de culpabilité dont elle tente de découvrir les causes en pratiquant la suspension du jugement (180), soit l'exploration critique, momentanément impartiale, du point de vue des victimes et de leurs bourreaux. Le parcours de cette strate est la condition de 
possibilité de l'acte de trancher et de juger: ce n'est pas elle, l'ancienne déportée, qui est coupable, ce sont ses bourreaux (181-182).

Cette étude de cas est le point de départ d'une montée en généralité qui tente de revisiter les dichotomies suivantes et de les penser sur un mode "continuiste" : personnel / public, individu / société, particulier / universel. Le témoignage de la rescapée répond en effet, aux yeux de Danblon, à un «besoin rationnel d'exprimer publiquement de tels jugements éthiques» (182-183). Il construit un espace psychologique privé, tout en formulant une exigence d'universalité de sujet qui " considère sa place au sein de l'humanité »; il y a " un regard inquiet sur l'humanité, cette nouvelle forme de prudence ». Le témoignage de l'ancienne déportée peut nous aider « à mieux penser l'action de juger dans les institutions contemporaines » (184). Il nous permet aussi de mieux comprendre le statut et les enjeux de la notion perelmanienne d'«auditoire universel» et de reconfigurer celle de sens commun (184-189).

15 Le titre du troisième et dernier chapitre, « la rhétorique ou l'art de rendre le monde humain ", définit les enjeux d'une technè qui " devrait fournir à la société un levier à l'art de vivre ». Danblon y présente trois domaines de la vie dans lesquels la rhétorique peut intervenir utilement, au prisme de la conception «naturaliste, humaniste et interdisciplinaire » (193) défendue dans cet ouvrage. Il s'agit de l'exercice individuel de la raison pratique, soit de la "rhétorique naturelle, présente chez chacun de nous " (193), puis du terrain des institutions publiques : «l'organisation de notre société, sa politique, son droit, son éducation » (ibid.) et enfin de "l'épistémologie des disciplines » (194-195). "Se réapproprier une raison rhétorique pleine et entière ", c'est donc voir dans la rhétorique un passeur entre les différents domaines de la raison et des savoirs humains (213), une pratique dynamique inhérente à la nature humaine et qui permet à l'homme de maîtriser son destin en « figurant le monde à sa mesure » (214).

Le moment est venu après avoir médité le discours de l'auteure sur la «suspension du jugement» et le rôle fondamental de la critique, mode de délibération et de problématisation qui se veut impartial - de pratiquer la critique à mon tour, mais une critique qui prend position puisque j'y évoquerai quelques points de désaccord.

L'ouvrage accorde une place fondamentale à la figuration, soit à l'action de « représenter » le monde où vit l'homme rhétorique et de le tailler à sa mesure (212 213). Il insiste tant sur cette activité où il est essentiellement question de " sensations ", de "pensées", d'"actions» et de "connaissances» présentées dans un éclairage "dynamique et jamais doctrinaire » (213) que l'on en vient à se demander où situer le jugement de valeur et les passions du désaccord. L'argumentaire oscille en fait entre l'apologie d'une culture rhétorique irénique, épurée et optimiste et la condamnation de ce qui est présenté comme son envers : l'esprit dogmatique, normatif et doctrinaire (145-146). L'approche « continuiste » défendue tout au long de l'ouvrage n'inclut donc pas ici celle défendue par Perelman pour lequel l'opposition dichotomique entre jugement de fait et jugement de valeur doit être dépassée, tout en étant fondamentale. Perelman et Olbrechts-Tyteca (1970 : 681-682) soulignent en effet à la fin du Traité « que la pratique et la théorie de l'argumentation sont corrélatives d'un rationalisme critique, qui transcende la dualité jugements de réalité - jugements de valeur, et rend les uns comme les autres solidaires de la personnalité du savant ou du philosophe, responsable de ses décisions dans le domaine de la connaissance comme dans celui de l'action » (je souligne). 

l'analyse du témoignage de l'ancienne déportée : « cette phase a ceci de remarquable qu'elle semble s'être installée comme première ressource de survie, ce qui peut se traduire par un refus éthique, au sens profond, d'intégrer une réalité qu'on trouve légitimement inacceptable. ». Ce passage est d'ailleurs suivi par une citation de Jean Améry où l'on peut lire : «La puissance morale de résistance renferme la protestation, la révolte contre le réel qui n'est raisonnable qu'aussi longtemps qu'il est moral» (ibid.). Danblon ne se prononce pas explicitement sur ce renoncement, délibéré ou non, à problématiser la question du rapport entre représentation et évaluation, taillage du réel référentiel et figuration de ce réel, marquée au sceau de croyances et de valeurs autres que le vrai, si bien que le lecteur est réduit à faire sa propre enquête dans les méandres de l'argumentaire. Cette déambulation en territoire épistémologique semble indiquer que le jugement de valeur renvoie, aux yeux de l'auteure, à une activité qui n'a rien de commun avec la rhétorique naturaliste et humaniste qu'elle défend: cette activité est synonyme de dogmatisme doctrinaire et renverrait à une normativité condamnable. On tenterait d'imposer des normes morales extérieures auxquelles on confierait artificiellement la déduction de ce qui est bien ou mal. L'homme s'en remettrait alors à son goût $d u$ "confort " (il serait plus facile de célébrer ou de condamner, de s'en remettre mécaniquement à des normes préétablies que de parcourir seul les diverses strates de la raison rhétorique -146); il ne résisterait pas dans ce cas à sa "propension » à renoncer à sa liberté et donc à ses responsabilités et s'alignerait sur des régularités préétablies. Perelman, cité à plusieurs reprises à des moments cruciaux de la démonstration (approche continuiste de la raison théorique et de la raison pratique, activation de la dissociation des notions afin de réaliser la suspension des jugements, théorisation de la notion d'« auditoire universel »), n'est pas sollicité sur ce point. La nouvelle rhétorique ouvre pourtant une troisième voie entre la neutralité et l'engagement normatif a priori : celle d'un engagement éthique qui se refuse à définir a priori le "bon» et le "mauvais " argument, reconnait les vertus heuristiques de la suspension du jugement qualifiée d'« impartialité » (1970: 79), mais la dissocie de l'«objectivité » située hors du champ de la rationalité rhétorique et accorde un rôle déterminant à la légitimation et à la validation du jugement du proposant par l'opposant.

Dernière remarque: aucun chercheur n'est contraint de traiter la question de l'engagement épistémologique et/ou éthique dans chacun de ses ouvrages et cela d'autant plus que Danblon assume explicitement la responsabilité de ses choix épistémologiques. Qu'il me soit donc juste permis de formuler le regret qu'aucun passage de L'homme rhétorique ne pratique la réflexivité ni ne prenne position quant à la question éthique formulée par Jean Améry citée plus haut, et cela d'autant plus que l'unique étude de cas effectuée ici est l'analyse du témoignage d'une rescapée de la Shoah. Danblon n'avait-elle pas écrit dans le numéro 9 d'Argumentation et Analyse du Discours (2012 : § 17):

Le choix - toujours tentant pour le chercheur - de ces débats à tonalité passionnée pose le problème des réactions émotionnelles que les données susciteront nécessairement chez le lecteur. [...] On a peine à croire, comme le note Marianne Doury à propos de corpus similaires, que le lecteur ne se range pas spontanément à l'analyse d'un chercheur qui dénoncerait un discours anti-démocrate comme "infâmant ». L'on voit ainsi les limites d'une analyse qui prend le risque de distribuer d'emblée les bons points 
et les mauvais points, non pas à partir d'une critique basée sur une expertise scientifique mais sur une position politique difficilement discutable pour les démocrates que nous sommes tous supposés être.

Doury (2004 : 152-153) s'opposait aussi, entre autres, dans le numéro (17) de Semen évoqué par Danblon, au cas de la " prise en otage de l'auditoire » confronté à un corpus consacré au récit de la Shoah par un survivant. Danblon veille, à chacune des étapes de l'analyse du récit de la rescapée, à rappeler explicitement les hypothèses de recherche pertinentes, à justifier son interprétation et à dire la victoire d'une raison rhétorique vivante incarnant le bien sur la cruauté du régime nazi, parangon du mal. N'est-ce pas la preuve que, contrairement à sa propre prise de position taxant a priori toute évaluation scientifique axiologique de normative et dogmatique, Danblon parvient à concilier « ouverture » épistémologique et engagement éthique humaniste, choix d'une étude de cas bouleversante et solidité d'une démonstration scientifique qui ne prend pas son auditoire « en otage»?

La brève discussion critique des points évoqués ici ne modifie aucunement les qualités de ce livre original qui ne peut que passionner tout lecteur à la recherche d'une conception innovatrice et puissante de la rhétorique, qui rend tout leur sens aux notions de technè et d'" homme complet ».

\section{BIBLIOGRAPHIE}

Charaudeau, Patrick. 2010. «Pour une interdisciplinarité "focalisée" dans les sciences humaines et sociales ", Questions de Communication 17, 195-222

Danblon, Emmanuelle. 2005. La fonction persuasive. Anthropologie du discours rhétorique : origines et actualité (Paris : Colin)

Danblon, Emmanuelle. 2012. « Il y a critique et critique : épistémologie des modèles d'argumentation » Argumentation et Analyse du Discours 9, [En ligne : http://aad.revues.org/ 1395]

Doury, Marianne. 2004. «La position du chercheur en argumentation », Semen 17, 149-163

Perelman, Chaïm \& Lucie Olbrechts- Tyteca. 1983. Le traité de l'argumentation La nouvelle rhétorique, 4 édition (Bruxelles : Éditions de l'Université de Bruxelles)

\section{AUTEURS}

\section{ROSELYNE KOREN}

Université Bar-Ilan, ADARR 